\title{
CARACTERÍSTICAS FISICOQUÍMICAS DE TRES VARIEDADES DE CAFÉ TOSTADO Y MOLIDO CULTIVADOS EN NORTE DE SANTANDER
}

\section{PHYSICOCHEMICAL CHARACTERISTICS OF THREE VARIETIES OF ROASTED AND GROUND COFFEE CULTIVATED IN NORTH OF SANTANDER}

\author{
${ }^{1}$ Parada O. Marisol, ${ }^{\star 1}$ Caballero P. Luz A. ${ }^{2}$ Rivera María E. \\ ${ }^{1}$ Universidad de Pamplona, Programa Ingeniería de Alimentos. Grupo de investigaciones GIBA. \\ ${ }^{2}$ Universidad de Pamplona, Departamento Civil y Ambiental. Km 1 el Buque, Pamplona, Norte de Santander, \\ Colombia. E-mail:kmari_05@hotmail.com,*luzcaballero@unipamplona.edu.co, maes@unipamplona.edu.co.
}

Recibido 15 de Febrero de 2017; Aceptado 30 de mayo de 2017.

\section{RESUMEN}

La calidad del café es el resultado de un conjunto de procesos que permiten el desarrollo y conservación de sus características físico-químicas hasta su transformación y consumo. El objetivo fue evaluar las características fisicoquímicas de tres variedades de café cultivados en el municipio de Toledo, Norte de Santander (Castillo, Colombia y Típica) de la especie Coffea arábiga. Las muestras de café (600 gramos) fueron recolectadas, descascarilladas, tostadas, molidas y empacadas; el tostado fue a $130 \pm 1{ }^{\circ} \mathrm{C}$, tamaño de partícula mediano (NTC 3534). Se realizaron pruebas de: $\mathrm{pH}$, humedad (NTC 2558), acidez titulable (NTC 5247), granulometría (NTC 3534) y color (NTC 3534). Los resultados fueron analizados estadísticamente con un ANOVA de un factor estableciendo si existían diferencias en 
Autor a quien dirigir la correspondencia: Caballero $\mathrm{P}$ Luz*, Correo electronico: luzcaballero@unipamplona.edu.co las características fisicoquímicas de las muestras de café. Las tres variedades de café presentaron diferencias estadísticas significativas entre el $\mathrm{pH}$ y el valor de $\mathrm{L}$ en el color.

Palabras clave: café, composición, tostado, variedad.

\section{ABSTRACT}

The quality of coffee is the result of a set of processes that allow the development and conservation of its physicalchemical characteristics until its transformation and consumption. The objective was to evaluate the physicochemical characteristics of three varieties of coffee cultivated in the municipality of Toledo, Norte de Santander (Castillo, Colombia and Típica) of the Coffea arábiga species. The coffee samples (600 grams) were collected, peeled, roasted, ground and packed; toasting was at $130 \pm 1^{\circ} \mathrm{C}$, medium particle size (NTC 3534). Tests were carried out: $\mathrm{pH}$, humidity (NTC 2558), titratable acidity (NTC 5247), granulometry (NTC 3534) and color (NTC 3534). The results were analyzed statistically with a one-way ANOVA, establishing whether there were differences in the physicochemical characteristics of the coffee samples. The three varieties of coffee showed significant statistical differences between the $\mathrm{pH}$ and the value of $\mathrm{L}$ in the color

Key words: coffee, composition, toasted, variety.

\section{INTRODUCCIÓN}

El café, además de ser una de las bebidas más populares en el mundo, es uno de los productos naturales de mayor complejidad, por su historia, su comercio y su riqueza química, conformada por Ácidos Orgánicos, Aldehídos, Cetonas, Esteres, Hidrocarburos de bajo peso molecular Aminoácidos, Cafeína, Carbohidratos, Proteínas, Trigonelina, Lípidos, Glucósidos y 
Minerales; cada uno de ellos contribuye con el sabor (más de cuatrocientos compuestos orgánicos e inorgánicos) y aroma característico (más de seiscientos compuestos) en su mayoría en concentraciones trazas.

El beneficio del café, es un proceso que consiste en retirar de las cerezas maduras las capas exteriores y dejar solo el café pergamino seco. Se realizan por vía húmeda o por vía seca (ver figura 1).

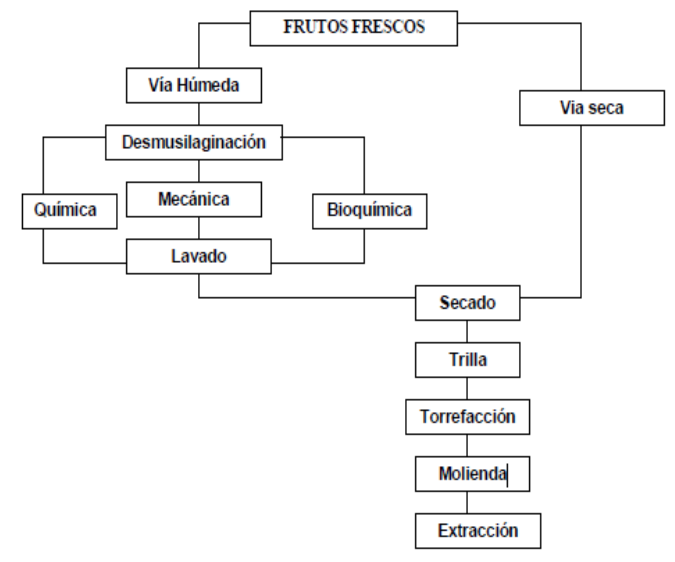

Figura 1. Diagrama de flujo para el beneficio del café por vía húmeda y por vía seca.

En la tostación se desarrollan diversas reacciones entre los componentes del grano de café almendra y se generan cientos de compuestos volátiles y sustancias, contenidos de ácidos clorogénicos totales en café almendra, la variedad y la madurez. La composición química de los granos cambia durante el proceso de tostado: el agua se disipa en el grano y una serie de reacciones químicas convierte los azúcares y almidones en aceites, los cuales otorgan al café gran parte de su aroma y sabor. Al ser tostado, el grano aumenta su tamaño al doble, y la caramelizarían del azúcar cambia el color de verde a marrón.El color y aspecto del grano tostado dependen del tiempo de tostado. Mientras más largo sea el tiempo de tostado, más oscuro será el grano. Generalmente el grano se tuesta durante 10 a 20 min a temperaturas oscilantes entre $400^{\circ} \mathrm{F}$ y $425^{\circ} \mathrm{F}$.

El secreto en el desarrollo del aroma y sabor del café reside en el tostado de los granos de café. El tiempo y la temperatura de tostado son cruciales en la preparación de una buena taza de café, así como en la determinación de qué características serán realizadas y cuáles serán variadas. Si el tostado es breve, los aceites no saldrán hasta la superficie y el café tendrá un sabor como de nuez y poco cuerpo.

Los granos tostados oscuros contienen menos acidez y un poco menos cafeína que los granos más claros, aunque también menor período de conservación, debido a la cantidad de aceites en la superficie. En los tostados más oscuros predomina el sabor ahumado, penetrante y quemado, ocultando el verdadero sabor del grano. Al contrario de las creencias populares, un tostado más oscuro no equivale a una taza de café más 
rica ni más fuerte. El tostado no determina si una taza de café será o menos fuerte, el factor determinante es la proporción de agua con respecto al café en el momento de prepararlo. Los granos tostados claros tienen un sabor más intenso, más altos en acidez que los tostados más oscuros. El café ha sido menos expuesto al calor, por lo cual las cualidades del grano son mejor mantenidas. Los tostados más claros se hacen con granos de más alta calidad, pues en este caso se expone el sabor real del grano (Puerta, G., 2011). Durante el proceso de tostión cambian los contenidos de los compuestos químicos y la concentración de éstos en los granos tostados con respecto al grano almendra: los polisacáridos disminuyen, la sacarosa se degrada completamente, los azúcares reductores aumentan, las proteínas disminuyen, los lípidos y la cafeína del grano tostado conforman cerca de la misma proporción o un poco más que en los granos almendra, la trigonelina disminuye, los ácidos aumentan, los ácidos clorogénicos disminuyen, las cenizas aumentan $Y$ las melanoidinas se crean. En la tostación, los ácidos clorogénicos se isomerizan, se unen a las melanoidinas, se hidrolizan, forman quinolactonas y se transforman en catecol, guayacol y pirogalol que tienen olores a humo y quemado. El contenido de ácidos clorogénicos en una taza de café depende de la especie, la madurez, el procesamiento y el grado de tostación.

El tueste del café es una fase vital dentro de su cadena de elaboración. El proceso de tostar los granos del café verde consiste en someterlos durante un tiempo limitado a una alta temperatura, intervalo durante el cual:

- Pierde peso, alrededor del 15/20\%, debido en gran parte a la evaporación de su humedad y en menor parte a la pirolisis de algunos componentes.

- El grano aumenta de volumen, entre un 100 y un $130 \%$ en el caso del café natural en función del tiempo de tueste y entre el 70 y el $80 \%$ para el torrefacto.

- Su color amarillo verdoso se transforma en un marrón, más o menos oscuro en función del grado de tueste escogido.

- La composición química del grano sufre una importante transformación, tanto a nivel cuantitativo como cualitativo. Azúcares, grasas, proteínas, substancias nitrogenadas no proteicas, ácidos, todo sufre una transformación debido a las altas temperaturas a que es sometido el grano.

Este último punto es el más interesante desde una óptica gastronómica pues es de ahí de donde surgen los aromas y sabores 
que han convertido al café en el rey de las infusiones.

Una vez tostado el café, el grano tostado se muele a diferentes granulometrías, dependiendo posteriormente del tipo de cafetera que se utilice para preparar la bebida. Los tres grados de molienda comercial son: grueso, medio y fino. Los productores recomiendan un molido grueso para cafetera percoladora, molido medio para cafeteras de filtro y el molido fino para preparar café tipo exprés.

El grado de espesor de la molienda tiene un impacto importante en el proceso de elaboración de la bebida, y es crítico saber combinar la consistencia del grado de fineza del café con el método de elaboración para poder extraer un sabor óptimo de los granos tostados. Los métodos de la elaboración del café que exponen la molienda de café a agua calentada durante mucho tiempo necesitan que las partículas tengan un mayor grosor que si, en cambio, se utilizan métodos más rápidos. Los granos que se muelen demasiado para un determinado método de elaboración expondrán demasiada área superficial al agua caliente y producirán un gusto amargo y áspero. En el otro extremo, si se muele poco y se dejan partículas excesivamente gruesas, se producirá un café débil, acuoso y falto de sabor (Café molido, 2014).

\section{MATERIALES Y MÉTODOS}

La evaluación fisicoquímica del café tostado y molido de las tres variedades se realizó por triplicado. Empleando como muestra patrón un café gourmet.

Se realizó un tostado manual de acuerdo a lo establecido por la NTC 3534 donde se pesaron $450 \mathrm{~g}$ de cada muestra, los granos de café estaban libres de impurezas y granos dañados. Los granos de café fueron sometidos a tostado en un sartén de acero inoxidable en una estufa eléctrica a temperatura de $130 \pm 1{ }^{\circ} \mathrm{C}$ aproximadamente, se tomó el tiempo de tostado hasta obtener el color deseado, seguidamente se removieron los granos de café constantemente con el fin de que el tostado fuera uniforme. Una vez terminado el tostado, se dejaron enfriar los granos tostados a temperatura ambiente $(21 \pm 1$ 
$\left.{ }^{\circ} \mathrm{C}\right)$. Inmediatamente se procedió a triturar los granos en un molino eléctrico marca WEGA, con un tamaño de partícula medianamente fina. Finalmente el café molido fue empacado en PET.

Se determinó el $\mathrm{pH}$, trabajando una relación $1: 10$, tomando1 $\mathrm{g}$ de café tostado y molido y $10 \mathrm{~mL}$ de agua destilada, introduciendo el $\mathrm{PH}$-metro y procediendo a realizar la lectura correspondiente.

Se determinó la acides titulable a las muestras de café tostado y molido de acuerdo a lo estipulado por la NTC 5247. Se colocaron $5 \mathrm{~g}$ de café tostado y molido con $100 \mathrm{ml}$ de agua a $90 \pm 1^{\circ} \mathrm{C}$ en un Erlenmeyer, se agito durante $3 \mathrm{~min}$, y se filtró inmediatamente sobre un papel filtro cualitativo; se lavó 3 veces el Erlenmeyer y el papel filtro con alícuotas de $5 \mathrm{ml}$ de agua destilada; el extracto se llevó a un volumen de $100 \mathrm{ml}$ en un balón aforado; a temperatura de $25 \pm 1{ }^{\circ} \mathrm{C}$. Posteriormente en un vaso de precipitado se agregaron 50 $\mathrm{ml}$ del extracto del café obtenido; con una pipeta volumétrica, se agito y se colocó el electrodo del potenciómetro previamente calibrado, se tituló potenciométricamente con el hidróxido de sodio valorado, agregando volúmenes de $0,1 \mathrm{ml}$ hasta el primer punto en que el pH llego a 6,5, este fue el punto de equivalencia, leyendo el volumen de Hidróxido de Sodio gastado para llegar a este punto, este fue el volumen de equivalencia. La acidez de la muestra se expresó en mg de ácido Clorogénicos en 1 $\mathrm{g}$ de café. Para obtener el contenido de acidez de la muestra se realizaron los siguientes cálculos:

\section{Acideztitulable $=$ vNaOHgastado*factordedilucion $*$ pesomol eculardeacidoclorogenico* 1 l $1000 l *$ (1000mg 1) / muestradecafe.}

La humedad se determinó según la NTC. 2558 empleando una balanza de humedad marca OHAUS. Se tomó una muestra de 3 g de cada una de las variedades a evaluar, posteriormente se colocaron en el platillo y se graduó la temperatura a $110 \pm 1^{\circ} \mathrm{C}$ con un tiempo de 30 minutos registrando la humedad final.

Se llevó a cabo la medición del color del café tostado molido de las tres variedades (Castillo, Colombia, y Típica), Por reflexión de la luz utilizando el Espectrofotómetro portátil X-Rite. Las medidas se tomaron en tres puntos distintos sobre la superficie del producto y por triplicado (NTC 3534), expresando los resultados de acuerdo al sistema de coordenadas CIELAB, $L^{*}, a^{*}, b^{*}$ (McLaren, 1980).

La prueba de calidad granulométrica del café tostado y molido se realizó de acuerdo 
a lo establecido por la NTC 3534, donde se pesaron $50 \mathrm{~g}$ de muestra, esta fue Introducida en el tamiz rotatorio dotado de 8 mallas que van desde $0,063 \mathrm{~mm}$ hasta 2 $\mathrm{mm}$ de luz de malla y se programó a una potencia de 9 , con 9 ciclos a un tiempo de
30 minutos. Donde se tuvo en cuenta la luz de malla, el peso del tamiz, masa de cernido, y la masa de rechazo para determina el tanto el porcentaje de cernido acumulado, como el porcentaje de cernido de rechazo.

\section{RESULTADOS Y DISCUSIÓN}

De acuerdo a la metodología planteada se obtuvieron los siguientes resultados: Las condiciones de las muestras empleadas en la cata de café fueron las mismas (procedencia de las muestras, proceso de Tostión y molienda (tiempo, temperatura y método)).

La Tabla 1 muestra los resultados de las pruebas fisicoquímicas $(\mathrm{pH}$, humedad y acidez) realizadas al café tostado y molido de las tres variedades (típica, Colombia, castillo) y Gourmet (muestra patrón) teniendo en cuenta que el $p$-valor $(0,00)$ es menor que el nivel de significancia $(\alpha=$ $0,05)$ existen diferencias estadísticamente significativas entre las características fisicoquímicas de las tres variedades estudiadas y la muestra patrón (café gourmet), por lo que fue necesario realizar el análisis de Post-Hoc para determinar en donde existían dichas diferencias (Tabla 1).
Tabla 1. Resultados del análisis fisicoquímico de las variedades (Típica, Colombia, Castillo) y Gourmet

\begin{tabular}{|l|c|c|c|}
\hline Variedad & $\begin{array}{c}\text { Humedad } \\
(\%)\end{array}$ & $\mathbf{p H}$ & $\begin{array}{c}\text { Acidez } \\
\text { titulable } \\
(\%)\end{array}$ \\
\hline \multirow{2}{*}{ Típica } & $3,25 \pm$ & $5,59 \pm$ & $4,03 \pm$ \\
& 0,0440 & 0,0502 & 0,0602 \\
\hline \multirow{3}{*}{ Colombia } & $3,04 \pm$ & $5,47 \pm$ & $3,96 \pm$ \\
& 0,0048 & 0,609 & 0,403 \\
\hline \multirow{3}{*}{ Castillo } & $3,05 \pm$ & $5,43 \pm$ & $3,98 \pm$ \\
& 0,0403 & 0,908 & 0,063 \\
\hline \multirow{3}{*}{ Gourmet } & $2,93 \pm$ & $4,64 \pm$ & $3,27 \pm$ \\
\hline \multirow{2}{*}{ Promedio } & 0,0079 & 0,0602 & 0,045 \\
\hline
\end{tabular}

Valor media \pm desviación estándar de los datos con $p$ valor $=0,000$ con un nivel de significancia del $5 \% n=3$. 
La Tabla 2 muestra que las diferencias estadísticas encontradas están en el pH con respecto a las otras características analizadas, evidenciando que las muestras de café del municipio de Toledo presentan características físico-químicas similares en las tres variedades, coincidiendo con similares resultados obtenidos por Lourdes, K, 2009.

Tabla 2. Resultados de las prueba Post-Hoc Tukey entre las características fisicoquímicas de las muestras estudiadas y la muestra patrón.

\begin{tabular}{|l|c|}
\hline \multicolumn{1}{|c|}{ Variable } & Valor \\
\hline Acidez (\%) & $3,88083^{\mathrm{a}}$ \\
\hline Humedad (\%) & $4,30417^{\mathrm{a}}$ \\
\hline $\mathrm{pH}$ & $5,23667^{\mathrm{b}}$ \\
\hline
\end{tabular}

Letras iguales no existen diferencias significativas al nivel de significancia del $5 \%$.

En la tabla 3 se evidencia que el parámetro L (luminosidad) presento diferencias estadísticamente significativas entre las variedades Castillo y las 3 muestras de café evaluadas, mientras que la variedad Colombia y Típica no presentaron diferencias entre ellas.

Todas las muestras de café presentaron diferencias en la Luminosidad $(L$ ) respecto a la muestra de café Gourmet.
Tabla 3. Resultados del color de las 4 muestras de café tostado y molido

\begin{tabular}{|l|c|c|c|}
\hline Variedades & Valor $^{*}$ & Valora $^{*}$ & Valor $^{*}$ \\
\hline Castillo & $\begin{array}{c}23,4 \pm \\
0,276^{\text {a }}\end{array}$ & $\begin{array}{c}34,4 \\
\pm 0,583^{\mathrm{a}}\end{array}$ & $\begin{array}{c}-22,4 \\
\pm 0,586^{\mathrm{a}}\end{array}$ \\
\hline Colombia & $22,5^{ \pm}$ & 35 & $-21,4$ \\
& $0,147^{\mathrm{b}}$ & $\pm 0,975^{\mathrm{a}}$ & $\pm 0,551^{\mathrm{a}}$ \\
\hline Típica & $22,1^{\mathrm{a}}$ & 30,4 & $-22 \pm$ \\
& $0,072^{\mathrm{b}}$ & $\pm 0,005^{\mathrm{a}}$ & $0,563^{\mathrm{a}}$ \\
\hline Gourmet & $18,72 \pm$ & $33,4 \pm$ & $21,9^{\mathrm{a}} \pm$ \\
(patrón) & $0,583^{\mathrm{c}}$ & $0,606^{\mathrm{a}}$ & $\pm 0,765^{\mathrm{a}}$ \\
\hline
\end{tabular}

Letras distintas indican diferencia significativa para $\mathrm{p}$ $\leq 0,05$.

Las tres muestras evaluadas no presentaron diferencias significativas en el color (valores a y b) respecto a la muestra patrón (café Gourmet) con valores promedio de $a=33,3$ y $b=22,0$.

La granulometría del café es un factor importan en la extracción de la infusión de café; a continuación se presentan los resultados obtenidos de la evaluación del grado de molienda de las tres variedades estudiadas. 


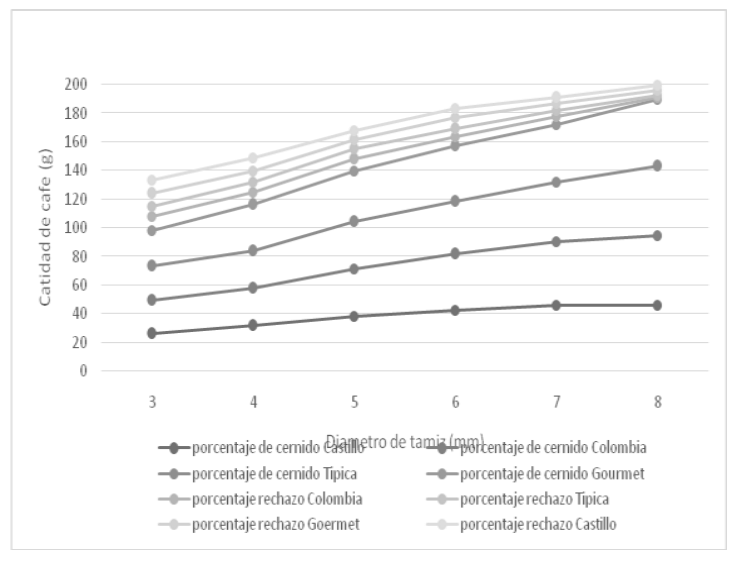

Figura 2. Resultados de la granulometría de las muestras de café tostado y molido

De la Figura 2 se infiere que las muestras analizadas presentan similitud en cuanto a la granulometría, debido a que el molido utilizado, el grado de tostión y las condiciones de tostión fueron las mismas para las cuatro muestras analizadas; sin embargo la variedad que mayor similitud presenta con la muestra patrón es la variedad Colombia.

De acuerdo a Puerta, G (1998), la granulometría del café es un factor importante para la extracción de componentes solubles y volátiles del café tostado. Una molienda muy fina dará un porcentaje alto de extracción con poca cantidad de agua el mismo resultado se obtendrá con una molienda más gruesa pero con más liquido con temperatura y presión elevada.

\section{CONCLUSIONES}

Las muestras de café tostado y molido de las tres variedades (Castillo, Colombia, y Típica) cultivadas en Toledo, Norte de Santander presentaron diferencias estadísticas significativas en el $\mathrm{pH}$ y la luminosidad (L).
Las muestras analizadas presentaron similitud en la granulometría, la variedad que mayor similitud presento con la muestra patrón fue la variedad Colombia. 


\section{REFERENCIAS BIBLIOGRAFICAS}

Caballero, P. Luz A., Ortega, Jessica C. y Maldonado, M. Lida Y. (2014). Evaluación del rendimiento de la extracción de café tostado molido comercial. Revista @limentech, Ciencia y Tecnología Alimentaria. ISSN 1692-7125. Volumen 12, N $\mathrm{N}^{\circ}$ 2, p. $23-31$.

CAFÉ MOLIDO. Consultado en: Septiembre 2014 en línea (http://www.nuestrocafe.com/opcion/cafe molido_17_75.php.

Cárdenas, E; Niltol, M; Figueredo, F; Moscoso, L; Zuñiga, F; Torres, G; Control de calidad del café. DESCO. Manual técnico.2003. Lima, Perú.

García B. Yulieth P., Caballero P. Luz A. y Maldonado O. Yohanna DC. (2016). Evaluación del color en el tostado de Haba (Vicia faba). Revista @limentech, Ciencia y Tecnología Alimentaria. ISSN 1692-7125. Volumen 14, $\mathrm{N}^{\circ} 2$, p. 53 66.

INSTITUTO COLOMBIANO DE NORMAS TECNICAS Y CERTIFICACIÓN, ICONTEC. NTC. 2558. café tostado y molido. Determinación del contenido de humedad. Método por determinación de la pérdida en masa a $103{ }^{\circ} \mathrm{C}$ (Método de
Rutina). Pág. 6. Primera Actualización. Octubre de 2000. Bogotá. D.C. Colombia.

INSTITUTO COLOMBIANO DE NORMAS TECNICAS Y CERTIFICACIÓN, ICONTEC. NTC. 3534. Café tostado o en grano molido. Determinación del grado de tostión. Pág. 6. Segunda Actualización. Diciembre de 2007. Bogotá. D.C. Colombia.

INSTITUTO COLOMBIANO DE NORMAS TECNICAS Y CERTIFICACIÓN, ICONTEC. NTC. 3534. Café tostado o en grano molido. Determinación de granulometría. Pág. 6. Segunda Actualización. Diciembre de 2007. Bogotá. D.C. Colombia.

INSTITUTO COLOMBIANO DE NORMAS TECNICAS Y CERTIFICACIÓN, ICONTEC. NTC. 5247. Café tostado en grano o molido. Determinación de la acidez títulable. Pág. 6. Editada en Marzo de 2004. Bogotá. D.C. Colombia.

Lourdes, K; Caracterización de cafés (Coffea arábiga) en los departamentos de Comayagua, El Paraíso, La Paz y Ocotepeque. 2009.

Mclaren, K. (1980). Food Colorimetry. In: Walford J, editor. Developments in food 
colours I. London, U.K.: Applied Science Publisher, Pág. 27-45.

Puerta, G; Factores de origen y proceso en la calidad y la química del café. Centro Nacional de Investigaciones de Café.
Cenicafé.

Chinchiná-Caldas-

Colombia.1998.

Puerta, G; Composición química de una taza de café. Programa de Investigación Científica. Fondo Nacional del Café. 2011. 